\title{
Hipoglucemia en pacientes con diabetes mellitus en los servicios de urgencias de dos hospitales de Bogotá, 2015-2017
}

\author{
Hypoglucemia in patients with diabetes mellitus in emergency \\ services of two hospitals in Bogotá, 2015-2017
}

Chaves $W^{1}$, Martínez $L^{2}$, Díaz $D^{3}$, Hernández $D^{3}$, Sanabria $M^{3}$.

${ }^{1}$ Médico Internista, profesor asistente, jefe del Departamento de Medicina Interna, Fundación Universitaria de Ciencias de la Salud, Sociedad de Cirugía de Bogotá, Hospital de San José. Bogotá, Colombia.

${ }^{2}$ Médico internista, instructor asistente, Departamento de Medicina Interna, Fundación Universitaria de Ciencias de la Salud, Sociedad de Cirugía de Bogotá, Hospital de San José, Bogotá, Colombia. ${ }^{3}$ Residente de Medicina Interna, Fundación Universitaria de Ciencias de la Salud, Sociedad de Cirugía de Bogotá, Hospital de San José. Bogotá, Colombia.

Autor de correspondencia:

Diana Alejandra Hernández Ramírez

Correo electrónico: dianaalejahr@gmail.com

Fecha de recepción: 23/01/2019

Fecha de aceptación: 21/10/2019

\section{Resumen}

Introducción: desde el punto de vista geno y fenotípico, la población diabética es heterogénea, lo que hace que algunos grupos especiales sean más susceptibles a complicaciones durante el tratamiento. Dentro de estas complicaciones, la hipoglucemia, es un motivo frecuente de consulta; sin embargo, la información acerca de episodios de esta alteración es limitada.

Objetivo: determinar las características sociodemográficas y clínicas de los pacientes diabéticos con hipoglucemia en los servicios de urgencias del Hospital de San José y el Hospital Infantil Universitario de San José.

Metodología: evaluación a una cohorte retrospectiva de pacientes diabéticos con hipoglucemia en los servicios de urgencias, durante enero de 2015 a diciembre de 2017. Se hizo un análisis descriptivo de los factores demográficos, clínicos y terapéuticos de esta población. Los datos fueron recolectados de las historias clínicas y las características fueron analizadas mediante estadística descriptiva.

Resultados: dentro de los datos encontrados, 201 pacientes presentaron hipoglucemia al ingreso (12,5\%). Con una mediana de 70 años, el 85,5 \% eran diabéticos tipo 2; de estos, $99 \%$ tenían manejo con insulina; con mayor frecuencia usaron insulina glargina $(58,1 \%)$ e insulina glulisina (36,3 \%). El 36,6 \% usó medicamentos orales, principalmente biguanidas; y la combinación más frecuente fue insulina basal más insulina de corta acción, en el 56,7 \%. La mediana de glucosa fue de 40,5 $\mathrm{mg} / \mathrm{dL}$ y la prueba de hemoglobina glicosilada (HbA1c) fue de 6,7 \%. La mortalidad hallada en esta población fue del 7,5 \%.

Conclusiones: la prevalencia de hipoglucemia en este estudio fue baja. Los resultados podrían sugerir la falta de individualización al momento de escoger los tratamientos y los objetivos terapéuticos, lo que refleja un aumento de los episodios de hipoglucemia grave. Por lo tanto, se debe realizar un tratamiento centrado en el paciente, considerando la presencia de episodios previos, los antecedentes de enfermedad cardiovascular, las enfermedades concomitantes asociadas, el peso, el costo de los medicamentos y la edad.

Palabras clave: hipoglucemia, diabetes mellitus, insulina, urgencias médicas.

\section{Abstract}

Introduction: from a genotypic and phenotypic point of view, the diabetic population is

heterogeneous, which makes some special groups more susceptible to complications during treatment. Among these complications, hypoglycemia is a frequent reason for consultation; however, information about episodes of this alteration is limited, especially in Latin America. 
Objective: to determine the sociodemographic and clinical characteristics of diabetic patients with hypoglycemia in the emergency services of the San José hospital and the San José University Children's hospital.

Methodology: retrospective evaluation of a cohort of diabetic patients with hypoglycemia in the emergency services from January 2015 to December 2017. A descriptive analysis of the demographic, clinical and therapeutic factors of this population was made. Data were collected from medical records and characteristics were analyzed using descriptive statistics.

Results: within the data found, 201 patients presented hypoglycemia at admission (12.5\%). With a median of 70 years, $85.5 \%$ were type 2 diabetics; of these, $99 \%$ had insulin management; more frequently they used insulin glargine (58.1\%) and insulin glulisine (36.3\%). 36.6\% used oral medications, mainly biguanides; and the most frequent combination was basal insulin plus short-acting insulin, in $56.7 \%$. The median glucose was $40.5 \mathrm{mg} / \mathrm{dL}$ and the glycosylated hemoglobin (HbA1c) test was 6.7\%. Mortality found in this population was $7.5 \%$.

Conclusions: the prevalence of hypoglycemia in this study was low. The results suggest that the lack of individualization when choosing treatments and therapeutic objectives, could be related with the increase in episodes of severe hypoglycemia. Therefore, a patient-centered treatment should be performed, considering the presence of previous episodes, the history of cardiovascular disease, associated concomitant diseases, weight, medication costs and age.

Keywords: Hypoglycemia, diabetes mellitus, insulin, emergencies.

\section{Introducción}

Desde el punto de vista geno y fenotípico, la población diabética es heterogénea. Factores como la ubicación geográfica, el acceso a los servicios de salud y el nivel educativo, impactan no solo su tratamiento, sino también sus complicaciones. Las guías actuales recomiendan el control estricto de la glucemia y su respectivo seguimiento con valores de hemoglobina glicosilada $^{(1)}$; sin embargo, teniendo en cuenta los factores mencionados, dicho control está estrechamente relacionado con el aumento en la incidencia de hipoglucemia ${ }^{(2)}$.

La consulta en los servicios de urgencias de pacientes diabéticos que presentan episodios de hipoglucemia es muy frecuente; sin embargo, la literatura encontrada en Latinoamérica sobre esta complicación es limitada. The Workgroup of the American Diabetes Association and The Endocrine Society recomienda que los pacientes deben ser alertados al encontrar niveles de glucemia menores de $70 \mathrm{mg} / \mathrm{dL}(3.9 \mathrm{mmol} / \mathrm{L})^{(3)}$. La hipoglucemia es una complicación grave, que se presenta con frecuencias similares en diabetes mellitus tipo 1 y 2 (DM1 y DM2 $)^{(4)}$. Se le atribuye al requerimiento de terapias de alta intensidad asociado con el uso de sulfonilureas, meglitinidas o insulina ${ }^{(3)}$; y puede ser fatal por el aumento en el riesgo de enfermedad cardiovascular ${ }^{(5-8)}$.

El riesgo de hipoglucemia en diabéticos se ha relaciona en mayor medida con el uso de insulinas y, en una menor frecuencia, con el uso de antidiabéticos orales, de estos los secretagogos, como la glibenclamida y la gliburida, son los más comunes ${ }^{(9)}$. El mayor consumo de insulinas y su tiempo de uso en la DM2 se ha relacionado con una mayor cantidad de eventos de hipoglucemia severa ${ }^{(7,10-12)}$.

Por otro lado, la hipoglucemia en la diabetes está asociada con un costo y una carga considerables para el servicio de salud; se estima, por ejemplo, que para el National Health Service (NHS) representa un costo anual de 39 millones de libras esterlinas $^{(13)}$. Desde el punto de vista clínico, la hipoglucemia está relacionada con el deterioro cognitivo y muerte asociada con episodios cardiovasculares ${ }^{(14,15)}$. Esta alteración tiene un impacto significativo en la calidad de vida de un individuo, su empleo, las interacciones sociales y las actividades cotidianas, como la conducción.

Además de estos efectos directos, la hipoglucemia puede tener un impacto indirecto sustancial en las consecuencias graves a largo plazo para la salud, ya que por temor a la aparición de nuevos eventos se pierde adherencia a los medicamentos, lo que lleva a un mal control glucémico ${ }^{(10,11)}$ y a la subsiguiente aparición de complicaciones micro y macrovasculares.

El objetivo de esta publicación es determinar las características sociodemográficas y clínicas en pacientes diabéticos con hipoglucemia que asistieron a los servicios de urgencias del Hospital de San José y el Hospital Infantil Universitario de San José, durante el período de enero de 2015 a diciembre de 2017.

\section{Métodos}

Se realizó una evaluación a una cohorte retrospectiva. Se incluyeron pacientes diabéticos que presentaron hipoglucemia, definida como glucosa sanguínea o capilar menor de 70 $\mathrm{mg} / \mathrm{dL}^{(3)}$, quienes asistieron a los servicios de urgencias de dos centros hospitalarios de tercer nivel de la ciudad de Bogotá, durante el período de enero de 2015 a diciembre de 2017. Los criterios de inclusión fueron: edad mayor de 18 años, antecedente conocido de diabetes mellitus tipo 1 o 2, e hipoglucemia al ingreso. Se excluyeron los pacientes con cáncer en estado terminal, gastrectomizados, embarazadas, intoxicados por alcohol y con historias clínicas incompletas. La recolección de los datos fue realizada por los investigadores.

De un total de 121.173 historias clínicas de pacientes a los que se les solicitó glucometría y glucemia al ingreso en el servicio de urgencias, se excluyeron a quienes tenían historias clínicas repetidas y se aplicaron filtros por los diagnósticos que tenían que ver con alteración de la conciencia, diabetes e hipoglucemia, para un total de 3.964. Al hacer la revisión de estas historias clínicas, se documentaron 1.598 pacientes diabéticos 
que consultaron el servicio de urgencias, y se incluyeron a los que presentaron hipoglucemia al ingreso.

Se realizó un análisis estadístico descriptivo; a las variables continuas se les aplicó la prueba de distribución de normalidad de Shapiro-Wilk y, con base en los resultados, se establecieron medidas de tendencia central (mediana) y de dispersión (rango intercuartílico). Las variables categóricas se analizaron y describieron en frecuencias absolutas y relativas. Finalmente, el procesamiento y análisis estadístico de la información recolectada se hizo en Stata $13^{\circledR}$ (College Station, Texas 77845 USA, Licensed to Fucsalud).

Esta investigación fue aprobada por los comités de ética en investigación de los centros hospitalarios y se acogió a las normas de Buenas Prácticas Clínicas en investigación. Esto garantizó la confidencialidad de la información en la recolección y análisis de los datos. Además, según la Resolución 8430 de 1993 de Colombia, el estudio fue clasificado como de riesgo mínimo.

\section{Resultados}

Durante el periodo comprendido entre enero de 2015 a diciembre de 2017, consultaron 1.598 pacientes diabéticos a urgencias; de estos, 201 presentaron hipoglucemia al ingreso, para una frecuencia de 12,5\%. Esta fue más frecuente en el sexo femenino $(54,2 \% ; n=109)$; la mediana de edad fue de 70 años; y la mayoría de los pacientes eran diabéticos tipo 2
(85,5 \%). Los síntomas más frecuentes reportados en las historias clínicas al ingreso fueron somnolencia $(50,7 \%)$ y diaforesis $(22,4 \%)$; y con muy baja frecuencia las palpitaciones y cefalea $(0,5 \%)$. Otras características de los pacientes se muestran en la Tabla 1.

De la población, el 83,5 \% se encontraba en manejo con insulinas. En el grupo de DM2, el $99 \%$ estaba con esta terapia y fue más frecuente el uso de glargina (58,1\%) seguido de la insulina NPH (protamina neutra de Hagedorn) (31\%). Para las insulinas de acción corta, la lispro y la glulisina se encontraron en 36,3 \% y 35,4 \%, respectivamente. El 36,6 \% recibían antidiabéticos orales, el 66,6\% usaban biguanidas y el 22,6\% sulfonilureas; también, se evidenció un bajo uso de inhibidores de la enzima dipeptidil peptidasa tipo 4 (DPP-4), análogos del péptido similar al glucagón tipo 1 (GLP1) y ninguno con inhibidores del cotransportador sodio-glucosa tipo 2 (SGLT2). La combinación más frecuente fue la de insulina basal más insulina de corta acción, seguida del uso de insulinas con biguanidas (Tabla 2).

La mediana de glucosa fue de 40,5 mg/dL (RIQ 30-53), siendo más baja en los diabéticos tipo 1 (Tabla 3). El tiempo de seguimiento de los pacientes fue hasta el egreso. El porcentaje de pacientes ingresados en la unidad de cuidados intensivos fue del $5 \%$. La mortalidad del total de la población fue del 7,5 \% y con una mediana de hospitalización de un día (mínimo 0 y máximo 27) (Tabla 4).

Tabla 1. Características sociodemográficas y clínicas de los pacientes diabéticos con hipoglucemia en urgencias en el Hospital de San José y el Hospital Infantil Universitario de San José (2015-2017)

\begin{tabular}{|c|c|c|c|}
\hline Características & Total $(n=201)$ & Mujeres $(n=109)$ & Hombres $(n=92)$ \\
\hline $\begin{array}{l}\text { Edad, años } \\
\text { Mediana, RIQ } \\
\text { Rango (min. - máx.) }\end{array}$ & $\begin{array}{c}70(59-80) \\
19-100\end{array}$ & $\begin{array}{c}73(60-81) \\
19-100\end{array}$ & $\begin{array}{c}66(58-80) \\
22-93\end{array}$ \\
\hline Hipertensión arterial, n (\%) & $150(75,7)$ & $81(75)$ & $69(76,6)$ \\
\hline $\begin{array}{l}\text { Tipo de diabetes (\%) } \\
\text { DM tipo } 1 \\
\text { DM tipo } 2\end{array}$ & $\begin{array}{c}29(14,4) \\
172(85,5)\end{array}$ & $\begin{array}{l}13(12) \\
96(88)\end{array}$ & $\begin{array}{l}16(17,3) \\
76(82,6)\end{array}$ \\
\hline $\begin{array}{l}\text { Tiempo diagnóstico de DM, } \\
\text { años } \\
\text { Mediana, RIQ } \\
\text { DM tipo } 1 \\
\text { DM tipo } 2\end{array}$ & $\begin{array}{c}14(5-20) \\
14,5(4,5-18,5) \\
12(6-20)\end{array}$ & $14,5(5-17,5)$ & $11(5-20)$ \\
\hline Retinopatía, n (\%) & $14(7)$ & $6(5,5)$ & $8(8,79)$ \\
\hline Enfermedad CV, n (\%) & $51(25,3)$ & $24(22)$ & $27(29,3)$ \\
\hline
\end{tabular}


Tabla 1. Características sociodemográficas y clínicas de los pacientes diabéticos con hipoglucemia en urgencias en el Hospital de San José y el Hospital Infantil Universitario de San José (2015-2017) (continuación)

\begin{tabular}{|c|c|c|c|}
\hline Características & Total $(n=201)$ & Mujeres $(n=109)$ & Hombres $(n=92)$ \\
\hline Nefropatía diabética n, (\%) & $69(34,3)$ & $26(23,8)$ & $43(46,7)$ \\
\hline DM tipo 1 & $4(13,8)$ & & \\
\hline DM tipo 2 & $65(37,8)$ & & \\
\hline $\mathrm{ECV}, \mathrm{n}(\%)$ & $27(13,4)$ & $12(11)$ & $15(16,3)$ \\
\hline Pie diabético, n (\%) & $8(4)$ & $1(0,9)$ & $7(7,6)$ \\
\hline Neuropatía diabética, n (\%) & $9(4,5)$ & $5(4,6)$ & $4(4,3)$ \\
\hline \multicolumn{4}{|l|}{ Síntomas, n (\%) } \\
\hline Palpitaciones & $1(0,5)$ & $1(0,9)$ & 0 \\
\hline Temblor & $6(3)$ & $3(2,7)$ & $3(3,2)$ \\
\hline Diaforesis & $45(22,4)$ & $28(25,7)$ & $17(18,4)$ \\
\hline Ansiedad & $2(1)$ & $2(1,8)$ & $0(0)$ \\
\hline Náuseas & $6(3)$ & $3(2,7)$ & $3(3,2)$ \\
\hline Confusión & $35(17,4)$ & $21(19,2)$ & $14(15,2)$ \\
\hline Somnolencia & $102(50,7)$ & $53(48,6)$ & $49(53,2)$ \\
\hline Alteración visual & $9(3)$ & $4(3,6)$ & $2(2,2)$ \\
\hline Alteración lenguaje & $12(6)$ & $4(3,7)$ & $8(8,9)$ \\
\hline Cefalea & $1(0,5)$ & 0 & $1(1,1)$ \\
\hline Mareo & $18(9)$ & $14(13)$ & $4(4.4)$ \\
\hline Otros* & $58(28,8)$ & $31(28,4)$ & $27(29,3)$ \\
\hline Ninguno & $7(3,5)$ & $2(1,8)$ & $5(5)$ \\
\hline
\end{tabular}

RIQ: rango intercuartílico; DM: diabetes mellitus; CV: cardiovascular; ECV: evento cerebrovascular. *Otros síntomas incluyen: convulsiones, dolor abdominal, emesis, disnea y dolor torácico.

Tabla 2. Tratamiento de los pacientes diabéticos con hipoglucemia a su ingreso en el servicio urgencias del Hospital de San José y el Hospital Infantil Universitario de San José (2015-2017)

\begin{tabular}{l|c|c|c|}
\hline \multicolumn{1}{c|}{ Características } & Total $(\mathbf{n = 2 0 1 )}$ & DM tipo 1 (n= 29) & DM tipo 2 (n= 172) \\
\hline Uso de insulina n (\%) & $165(82)$ & $27(93)$ & $171(99)$ \\
\hline Insulina de larga acción n (\%) & & & $40(32)$ \\
NPH & $46(31)$ & $6(26)$ & $73(58,4)$ \\
Glargina & $86(58,1)$ & $13(56,5)$ & $12(9,6)$ \\
Detemir & $15(10,1)$ & $3(13)$ & $0(0)$ \\
Degludec & $1(0,6)$ & $1(4,3)$ & $25(28,1)$ \\
\hline Insulina de corta acción n (\%) & & $3(14,2)$ & $34(38,2)$ \\
Cristalina & $28(25,4)$ & $6(28,5)$ & $27(30,4)$ \\
Lispro & $40(36,3)$ & $12(57,1)$ & $3(3,3)$ \\
Glulisina & $39(35,4)$ & $0(0)$ & $68(41,2)$ \\
Aspart & $3(2,7)$ & $1(4,1)$ & \\
\hline Uso de AO $n$ (\%) & $69(36,6)$ & & \\
\hline
\end{tabular}


Tabla 2. Tratamiento de los pacientes diabéticos con hipoglucemia a su ingreso en el servicio urgencias del Hospital de San José y el Hospital Infantil Universitario de San José (2015-2017) (continuación)

\section{Características}

Tipo de AO n (\%)

Biguanidas

Sulfonilureas

Tiazolidinedionas

Glinidas

Inhibidores alfa1 glucosidasa

Análogos GLP1

Inh. DPP4

Inh. SGLT2

Tipo combinaciones $\mathrm{n}(\%)$

Ins. xorta + Ins. larga

Insulinas + biguanida

Biguanida + sulfonilurea

Biguanida + Inh. DPP4

Insulina + Inh. DPP4

Insulina + biguanida + Inh. DPP4

Insulina + GLP1

Inh. SGLT2 + biguanida

Total $(n=201)$

DM tipo $1(n=29)$

DM tipo $2(n=172)$

AO: antidiabéticos orales; Ins.: insulinas; Inh.: inhibidor.

Tabla 3. Laboratorios y función renal al ingreso de los pacientes diabéticos con hipoglucemia en los servicios de urgencias del Hospital de San José y el Hospital Infantil Universitario de San José (2015- 2017)

\section{Características}

Total $(\mathbf{n}=\mathbf{2 0 1})$

DM tipo $1(n=29)$

DM tipo $2(n=172)$

\begin{tabular}{|c|c|c|c|}
\hline $\begin{array}{l}\text { Glucosa de ingreso } \\
\text { Mediana, RIQ }\end{array}$ & $40,5(30-53)$ & $37(24-53)$ & $42(30-53)$ \\
\hline $\begin{array}{l}\text { HbA1c } \\
\text { Mediana, RIQ }\end{array}$ & $6,7(5,9-8,2)$ & $7,57(5,5-14)$ & $6,7(5,9-8,2)$ \\
\hline $\begin{array}{l}\text { BUN (mg/dL) } \\
\text { Mediana, RIQ }\end{array}$ & $22,5(15,8-38)$ & $14,5(13-18)$ & $24(17-4)$ \\
\hline $\begin{array}{l}\text { Creatinina }(\mathrm{mg} / \mathrm{dL}) \\
\text { Mediana, RIQ }\end{array}$ & $0,8(0,7-1,7)$ & $0,8(0,7-0,84)$ & $0,9(0,8-2)$ \\
\hline $\begin{array}{l}\text { TFG por CKD/EP } \\
\text { Mediana, RIQ }\end{array}$ & $73,4(33,2-88,2)$ & $113,4(84,9-124)$ & $68(27,8-84,6)$ \\
\hline Estadios de falla renal $n,(\%)$ & & & \\
\hline 1 & $40(21,4)$ & $16(72,7)$ & $24(14,5)$ \\
\hline 2 & $72(38,5)$ & $4(18,1)$ & $68(41,2)$ \\
\hline 3 & $31(16,5)$ & $2(9)$ & $29(17,5)$ \\
\hline 4 & $18(9,6)$ & $0(0)$ & $18(10,9)$ \\
\hline 5 & $26(14)$ & $0(0)$ & $26(15,7)$ \\
\hline
\end{tabular}

HbA1c: hemoglobina glicosilada; TFG: tasa de filtración glomerular; RIQ: rango intercuartílico. 
Tabla 4. Líquidos intravenosos recibidos al ingreso y desenlaces hospitalarios de los pacientes diabéticos con hipoglucemia en los servicios de urgencias del Hospital de San José y el Hospital Infantil Universitario de San José (2015-2017)

\begin{tabular}{l|c|c|c|}
\hline \multicolumn{1}{|c|}{ Características } & Total $(\mathbf{n}=\mathbf{2 0 1})$ & DM tipo $\mathbf{1}(\mathbf{n}=\mathbf{2 9})$ & DM tipo 2 $(\mathbf{n}=\mathbf{1 7 2})$ \\
\hline LEV $\mathbf{n}(\%)$ & $178(89,4)$ & $26(92,8)$ & $152(88,9)$ \\
\hline Tipo de LEV (\%) & & & \\
SSN & $11(6,2)$ & $3(11,1)$ & $8(5,3)$ \\
LR & $7(4)$ & $1(3,7)$ & $6(3,9)$ \\
DAD 5\% & $21(11,8)$ & $2(7,4)$ & $19(12,5)$ \\
DAD 10\% & $136(76,4)$ & $21(77,7)$ & $115(76,1)$ \\
Combinación con DAD & $3(1,7)$ & $0(0)$ & $3(1)$ \\
\hline UCI n (\%) & $10(5)$ & $0(0)$ & $10(5,8)$ \\
\hline Tiempo hospitalización (días) & $1(0-2)$ & $1(0-1)$ & $1(0-2)$ \\
Mediana, RIQ & $0-27$ & $1(3,5)$ & $14(8,1)$ \\
\hline Mínimo - máximo & $15(7,5)$ & & \\
\hline Muerte n, (\%) & & & \\
\hline
\end{tabular}

LEV: líquidos intravenosos; SSN: solución salina normal; LR: lactato de Ringer; DAD: dextrosa en agua destilada.

\section{Discusión}

En el mundo la proporción de pacientes diabéticos con hipoglucemia es variable; llega a ser tan baja como del $3 \%{ }^{(16)} \mathrm{y}$ tan elevada como del $41 \%-46 \%{ }^{(17,18)}$, en comparación con lo encontrando en nuestro estudio del 12,5 \%. En Latinoamérica la frecuencia es baja en el servicio de urgencias por todas las causas, como lo reportado por Vargas y colaboradores en un estudio realizado en Chile $(0,14 \%)^{(19)}$, lo cual es similar a lo hallado en nuestro estudio $(0,18 \%)$.

Los reportes en Latinoamérica, específicamente en la población de diabéticos, son altos en comparación con otros países; alcanzan el $72 \%$ para DM1, como lo reportado por The global HAT study ${ }^{(20)}$, Emral y colaboradores ${ }^{(2)}$, y Donnelly y colaboradores ${ }^{(16)}$. En nuestro estudio la presencia de hipoglucemia fue más alta en los diabéticos tipo 2, lo que puede ser explicado por la longevidad, el uso de insulinas en el $99 \%$, la presencia de enfermedad renal en estadios 2, 3, 4 y 5 en el $73 \%$, objetivos terapéuticos en valores de hemoglobina glicosilada estrictos menores del 7 \% para nuestra población de pacientes y mayor tiempo desde el diagnóstico. Todo lo anterior podría sugerir mayor falla en la célula beta, lo que aumenta los requerimientos de insulina, así como el tipo y variabilidad de esta ${ }^{(4,16,20)}$; todos estos factores de riesgo son ampliamente identificados para el desarrollo de hipoglucemia ${ }^{(21-23)}$.

En los diferentes estudios no hay una asociación entre los niveles de glucosa y el tipo de síntoma ${ }^{(24)}$. La presentación clínica es variable; en este estudio los síntomas neuroglucopénicos, principalmente la alteración de la conciencia y confusión, fueron los más frecuentes. Contrario a estos hallazgos, algunos estudios reportan frecuencias más elevadas de síntomas autonómicos, como diaforesis, temblor y fatiga ${ }^{(25)}$. Esto depende de la forma de recolección de la información, ya que algunos estudios en población de DM lo hacen con cuestionarios ${ }^{(26,27)}$. En estos los pacientes identifican síntomas tempranos ambulatoriamente no reportados en las consultas de seguimiento y llegan a acostumbrarse a síntomas que son inespecíficos o no reconocidos, dejando pasar estas alarmas previas a la presentación de un cuadro grave de hipoglucemia ${ }^{(2)}$, como principal factor de riesgo para la recurrencia de este con más severidad y mayor mortalidad ${ }^{(5)}$.

El uso de insulinas se ha asociado con un mayor número de episodios de hipoglucemia, lo que depende de las características del paciente, el tipo de insulina, su variabilidad, combinaciones y esquema utilizado ${ }^{(22,24)}$. En este estudio se observó una mayor frecuencia de hipoglucemias con el uso de insulinas de larga acción, glargina frente acción intermedia NPH, diferente a los hallazgos reportados por Rosenstock y colaboradores, quienes encontraron un menor número de episodios de hipoglucemia severa (odds ratio [OR] 0,74; p = 0,030), cualquier evento grave (OR 0,64; $\mathrm{p}=0,035)$ y una reducción en las probabilidades de hipoglucemia diurna y severa del $26 \%$ y $36 \%$ con glargina ${ }^{(28)}$.

Contradictorio a lo expuesto, se publicó recientemente (2018) un estudio observacional retrospectivo de pacientes con diabetes tipo 2, a quienes se les inició un esquema basal con análogos de insulina frente a NPH, entre el 2006 y el 2015. 
Durante un seguimiento de 1,7 años, hubo 39 visitas al hospital relacionadas con la hipoglucemia o ingresos hospitalarios entre 1928 pacientes que iniciaron análogos de insulina (11,9 eventos [IC 95\%: 8,1-15,6] por 1000 personas-año), en comparación con 354 visitas al hospital o ingresos hospitalarios relacionados con hipoglucemias entre 23.561 pacientes que iniciaron insulina NPH (8,8 eventos [IC $95 \%$ : 7,9-9,8] por 1.000 persona-año) (diferencia entre grupos: 3,1 eventos [IC del $95 \%$ : -1,5-7,7] por 1.000 persona-año; $p=0,07)^{(29)}$. Estos resultados, que se encuentran en relación con los hallazgos del presente estudio, sugieren que el uso de análogos de insulina de tipo glargina podría no tener ventajas adicionales sobre el riesgo de hipoglucemia, disminución de las visitas a urgencias y hospitalizaciones por hipoglucemia.

En nuestro estudio, la combinación de insulina con medicamentos antidiabéticos fue mayor con biguanidas que con sulfonilureas; y fue bajo el uso de otros, como inhibidores de DPP-4, análogos del GLP1 y antagonistas de SGLT2. Para estos últimos, estudios han demostrado una menor frecuencia de hipoglucemia y beneficios cardiovasculares ${ }^{(30-33)}$.

Roumie y su equipo de investigadores encontraron un mayor número de eventos de hipoglucemia en una cohorte retrospectiva de pacientes en tratamiento con metformina, a quienes se les inició insulina o sulfonilureas, con una intensificación progresiva del tratamiento durante 14 meses. Se presentaron 30,9 frente a 24,6 eventos por 1000 persona-año de hipoglucemia (hazard ratio [HR]: 1,30 [IC $95 \%$ : 1,06-1,59]), y para recurrencia de hipoglucemia 39,1 frente a 30,0 por 1000 personas-año (HR: 1,39; [IC del 95 \%: 1,12-1,72] en la combinación metformina más insulina ${ }^{(34)}$, similar a los datos reportados en este estudio.

Los reportes sobre mortalidad, eventos cardiovasculares e hipoglucemia severa se han establecido en estudios previos ${ }^{(5)}$. Esta última se ha asociado con la mortalidad por todas las causas en DM1 (HR: 1,98 [IC 95 \%: 1,25-3,17]) y DM2 (HR: 1,74 [IC $95 \%: 1,39-2,18$ ], sin enfermedad cardiovascular y con enfermedad cardiovascular (HR: 2,03 [IC $95 \%$ : 1,66-2,47] en DM1 y HR: 2,48 [IC 95 \%: 2,21-2,79] en DM2) ${ }^{(6)}$.

En 2010, un análisis epidemiológico retrospectivo del $A C$ CORD trial buscó determinar la asociación entre la hipoglucemia y la mortalidad de pacientes con DM2 con niveles de HbA1c de 7,5 \% o más, con enfermedad cardiovascular clínica o subclínica y dos factores de riesgo cardiovascular con intervención intensiva $\mathrm{HbA1c}<6 \%$ o estándar HbA1c 7 a 7,4 \%, glucosa $<50 \mathrm{mg}$ o que los síntomas se resolvieran con el tratamiento y asistencia de otra persona. En el análisis se evaluaron todas las causas de mortalidad o la mortalidad específica, la cual fue del 2,8 \% en quienes presentaron uno o más episodios de hipoglucemia o requirieron asistencia, en comparación con el 1,2 \% de los que no presentaron eventos o no requirieron asistencia (HR: 1,41 [IC 95 \%: 1,03-1,93]). Los investigadores concluyeron que la hipoglucemia severa sintomática se asoció con un incremento en el riesgo de muerte en cada brazo del estudio, sin diferencias en la mortalidad entre el tratamiento intensivo frente al estándar ${ }^{(8)}$.

En otros estudios se encontró una mortalidad de 0,32 \% ${ }^{(36)}$, menor que en la población de la presente investigación. Los hallazgos fueron similares, en especial en el grupo de los diabéticos tipo 2 con mayor severidad del episodio, hospitalización, ingreso a la unidad de cuidados intensivos y mortalidad explicable por las características ya descritas de este grupo de pacientes ${ }^{(19)}$.

Las guías de la Sociedad Americana de Endocrinólogos recomiendan metas de hemoglobina glicosilada considerando el riesgo de hipoglucemia, y factores como la enfermedad cardiovascular, el peso, el costo y las preferencias del paciente, por lo que se debe individualizar el tratamiento evaluando los riesgos y beneficios, así como los objetivos terapéuticos centrados en el paciente ${ }^{(9,35)}$.

Este es el primer estudio hecho en Colombia sobre hipoglucemia como descompensación aguda en pacientes diabéticos que consultan a los servicios de urgencias; además, tiene en cuenta los síntomas y las diferentes combinaciones de medicamentos. Entre las debilidades de la presente investigación se encuentra la naturaleza retrospectiva del estudio y que no cuenta con un sistema de grupo relacionado por el diagnóstico. Esto dificulta la recolección de la información y explica la falta de datos en las historias clínicas.

\section{Conclusión}

En este estudio la prevalencia de hipoglucemia fue baja. Los resultados podrían sugerir la falta de individualización al momento de escoger los tratamientos y los objetivos terapéuticos, lo que refleja un aumento de los episodios de hipoglucemia grave. Por lo tanto, se debe realizar un tratamiento centrado en el paciente, considerando la presencia de episodios previos, los antecedentes de enfermedad cardiovascular, las enfermedades concomitantes asociadas, el peso, el costo de los medicamentos y la edad.

\section{Agradecimientos}

Agradecemos al servicio de Medicina Interna y a la División de Investigaciones del Hospital de San José, al Hospital Infantil Universitario de San José y a la Fundación Universitaria de Ciencias de la Salud, por el apoyo y colaboración para llevar a cabo este trabajo.

\section{Financiación}

Financiación del estudio realizada por los autores.

\section{Conflicto de intereses}

Posibles conflictos de interés. 


\section{Referencias}

1. American Diabetes Association. 8. Pharmacologic Approaches to Glycemic Treatment: Standards of Medical Care in Diabetes-2018. Diabetes Care. 2018;41(Suppl 1):S73-85.

2. Emral R, Pathan F, Cortés CAY, El-Hefnawy MH, Goh S-Y, Gómez AM, et al. Self-reported hypoglycemia in insulin-treated patients with diabetes: Results from an international survey on 7289 patients from nine countries. Diabetes Res Clin Pract. 2017;134:17-28.

3. Seaquist ER, Anderson J, Childs B, Cryer P, Dagogo-Jack S, Fish L, et al. Hypoglycemia and diabetes: a report of a workgroup of the American Diabetes Association and the Endocrine Society. Diabetes Care. 2013;36(5):1384-95.

4. Leese GP, Wang J, Broomhall J, Kelly P, Marsden A, Morrison W, et al. Frequency of severe hypoglycemia requiring emergency treatment in type 1 and type 2 diabetes: a population-based study of health service resource use. Diabetes Care. 2003;26(4):1176-80.

5. Bonds DE, Miller ME, Bergenstal RM, Buse JB, Byington RP, Cutler JA, et al. The association between symptomatic, severe hypoglycaemia and mortality in type 2 diabetes: retrospective epidemiological analysis of the ACCORD study. BMJ. 2010;340:b4909.

6. Khunti K, Davies M, Majeed A, Thorsted BL, Wolden ML, Paul SK. Hypoglycemia and risk of cardiovascular disease and all-cause mortality in insulin-treated people with type 1 and type 2 diabetes: a cohort study. Diabetes Care. 2015;38(2):316-22.

7. Frier BM. Hypoglycaemia in diabetes mellitus: epidemiology and clinical implications. Nat Rev Endocrinol. 2014;10(12):711-22.

8. Action to Control Cardiovascular Risk in Diabetes Study Group, Gerstein HC, Miller ME, Byington RP, Goff DC, Bigger JT, et al. Effects of intensive glucose lowering in type 2 diabetes. N Engl J Med. 2008;358(24):2545-59.

9. Mathur S, Zammitt NN. Optimal glycaemic control in elderly people with type 2 diabetes: what does the evidence say?. Drug Safety BM. 2015;38(1).

10. Brito-Sanfiel M, Diago-Cabezudo J, Calderon A. Economic impact of hypoglycemia on healthcare in Spain. Expert Rev Pharmacoecon Outcomes Res. 2010;10(6):649-60.

11. Edridge CL, Dunkley AJ, Bodicoat DH, Rose TC, Gray LJ, Davies MJ, et al. Prevalence and Incidence of Hypoglycaemia in 532,542 People with Type 2 Diabetes on Oral Therapies and Insulin: A Systematic Review and MetaAnalysis of Population Based Studies. PloS One. 2015;10(6):e0126427.

12. Cryer PE, Axelrod L, Grossman AB, Heller SR, Montori VM, Seaquist ER et al. Evaluation and management of adult hypoglycemic disorders: an Endocrine Society Clinical Practice Guideline. J Clin Endocrinol Metab. marzo de 2009;94(3):709-28.

13. Hex N, Bartlett C, Wright D, Taylor M, Varley D. Estimating the current and future costs of Type 1 and Type 2 diabetes in the UK, including direct health costs and indirect societal and productivity costs. Diabet Med J Br Diabet Assoc. 2012;29(7):855-62.

14. Zoungas S, Patel A, Chalmers J, de Galan BE, Li Q, Billot L, et al. Severe Hypoglycemia and Risks of Vascular Events and Death. N Engl J Med. 2010;363(15):1410-8.

15. Feinkohl I, Aung PP, Keller M, Robertson CM, Morling JR, McLachlan S et al. Severe hypoglycemia and cognitive decline in older people with type 2 diabetes: the Edinburgh type 2 diabetes study. Diabetes Care. 2014;37(2):507-15.

16. Donnelly LA, Morris AD, Frier BM, Ellis JD, Donnan PT, Durrant R, et al. Frequency and predictors of hypoglycaemia in Type 1 and insulin-treated Type 2 diabetes: a population-based study. Diabet Med J Br Diabet Assoc. 2005;22(6):749-55.

17. UK Hypoglycaemia Study Group. Risk of hypoglycaemia in types 1 and 2 diabetes: effects of treatment modalities and their duration. Diabetologia. 2007;50(6):1140-7.

18. ter Braak EW, Appelman AM, van de Laak M, Stolk RP, van Haeften TW, Erkelens DW. Clinical characteristics of type 1 diabetic patients with and without severe hypoglycemia. Diabetes Care. 2000;23(10):1467-71.

19. Cecilia VR, San Cristóbal F, Jara P, López S, Trujillo J, Cecilia VR, et al Caracterización de eventos de hipoglicemia en pacientes diabéticos y no diabéticos atendidos en un servicio de urgencia. Rev Médica Chile. 2017;145(11):1387-93
20. Khunti K, Alsifri S, Aronson R, Cigrovski Berković M, Enters-Weijnen C, Forsén T, et al. Rates and predictors of hypoglycaemia in 27585 people from 24 countries with insulin-treated type 1 and type 2 diabetes: the global HAT study. Diabetes Obes Metab. 2016;18(9):907-15.

21. Papademetriou V, Lovato L, Doumas M, Nylen E, Mottl A, Cohen RM, et al Chronic kidney disease and intensive glycemic control increase cardiovascular risk in patients with type 2 diabetes. Kidney Int. 2015;87(3):649-59.

22. ORIGIN Trial Investigators. Predictors of nonsevere and severe hypoglycemia during glucose-lowering treatment with insulin glargine or standard drugs in the ORIGIN trial. Diabetes Care. 2015;38(1):22-8.

23. Gubitosi-Klug RA, Braffett BH, White NH, Sherwin RS, Service FJ, Lachin JM, et al. Risk of Severe Hypoglycemia in Type 1 Diabetes Over 30 Years of Follow-up in the DCCT/EDIC Study. Diabetes Care. 2017;40(8):1010-6.

24. McAulay V, Deary IJ, Frier BM. Symptoms of hypoglycaemia in people with diabetes. Diabet Med J Br Diabet Assoc. 2001;18(9):690-705.

25. Hepburn DA, MacLeod KM, Pell AC, Scougal IJ, Frier BM. Frequency and symptoms of hypoglycaemia experienced by patients with type 2 diabetes treated with insulin. Diabet Med J Br Diabet Assoc. 1993;10(3):231-7.

26. Östenson CG, Geelhoed-Duijvestijn P, Lahtela J, Weitgasser R, Markert Jensen M, Pedersen-Bjergaard U. Self-reported non-severe hypoglycaemic events in Europe. Diabet Med J Br Diabet Assoc. 2014;31(1):92-101.

27. Orozco-Beltrán D, Artola S, Jansà M, Lopez de la Torre-Casares M, Fuster E. Impact of hypoglycemic episodes on health-related quality of life of type-2 diabetes mellitus patients: development and validation of a specific QoLHYPOC questionnaire. Health Qual Life Outcomes. 2018;16(1):52.

28. Rosenstock J, Fonseca V, Schinzel S, Dain M-P, Mullins P, Riddle M. Reduced risk of hypoglycemia with once-daily glargine versus twice-daily NPH and number needed to harm with NPH to demonstrate the risk of one additional hypoglycemic event in type 2 diabetes: Evidence from a long-term controlled trial. J Diabetes Complications. 2014;28(5):742-9.

29. Lipska KJ, Parker MM, Moffet HH, Huang ES, Karter AJ. Association of Initiation of Basal Insulin Analogs vs Neutral Protamine Hagedorn Insulin With Hypoglycemia-Related Emergency Department Visits or Hospital Admissions and With Glycemic Control in Patients With Type 2 Diabetes. JAMA. 2018;320(1):53-62.

30. Zinman B, Wanner C, Lachin JM, Fitchett D, Bluhmki E, Hantel S, et al. Em pagliflozin, Cardiovascular Outcomes, and Mortality in Type 2 Diabetes. N Engl J Med. 2015;373(22):2117-28.

31. Marso SP, Poulter NR, Nissen SE, Nauck MA, Zinman B, Daniels GH, et al Design of the liraglutide effect and action in diabetes: evaluation of cardiovascular outcome results (LEADER) trial. Am Heart J. 2013;166(5):823830.e5.

32. Fulcher G, Matthews DR, Perkovic V, de Zeeuw D, Mahaffey KW, Mathieu $\mathrm{C}$, et al. Efficacy and safety of canagliflozin when used in conjunction with incretin-mimetic therapy in patients with type 2 diabetes. Diabetes Obes Metab. 2016;18(1):82-91.

33. Scirica BM, Bhatt DL, Braunwald E, Steg PG, Davidson J, Hirshberg B, et al The design and rationale of the saxagliptin assessment of vascular outcomes recorded in patients with diabetes mellitus-thrombolysis in myocardial infarction (SAVOR-TIMI) 53 study. Am Heart J. 2011;162(5):818-825.e6.

34. Roumie CL, Min JY, Greevy RA, Grijalva CG, Hung AM, Liu X, et al. Risk of hypoglycemia following intensification of metformin treatment with insulin versus sulfonylurea. CMAJ Can Med Assoc J J Assoc Medicale Can 2016;188(6):E104-12

35. Davies MJ, D’Alessio DA, Fradkin J, Kernan WN, Mathieu C, Mingrone G, et al. Management of hyperglycaemia in type 2 diabetes, 2018. A consensus report by the American Diabetes Association (ADA) and the European Association for the Study of Diabetes (EASD). Diabetologia. 2018;61(12):2461-98

36. Barranco RJ, Gomez-Peralta F, Abreu C, Delgado M, Palomares R, Romero F, et al. Incidence and care-related costs of severe hypoglycaemia requiring emergency treatment in Andalusia (Spain): the PAUEPAD project. Diabet Med J Br Diabet Assoc. 2015;32(11):1520-6. 